Low $f$ requency spect $r$ a of the hexamet hyl benzene / t et racyanoet hyl ene el ectron donor accept or compl exes i $n$ sol ut $i$ on st udi ed by terahert $z$ t i me- domai $n$ spect r oscopy

\begin{tabular}{|l|l|}
\hline 著者 & $\begin{array}{l}\text { YAMAMOTO Kohj i , KABI R Md. Humayun, HAYASH } \\
\text { M chi t oshi , TOM NAGA Kei suke }\end{array}$ \\
\hline $\begin{array}{l}\text { j our nal or } \\
\text { publ i cat i on t i t l e }\end{array}$ & Physi cal chemi st ry, chem cal physi cs \\
\hline vol une & 7 \\
\hline number & 9 \\
\hline page $\mathrm{r}$ ange & $1945-1952$ \\
\hline year & $2005-04$ \\
\hline URL & ht t p: //hdl . handl e. net /10098/3034 \\
\hline
\end{tabular}




\title{
Low-frequency spectra of the hexamethylbenzene/ tetracyanoethylene electron donor-acceptor complexes in solution studied by terahertz time-domain spectroscopy
}

\author{
Kohji Yamamoto, $\dagger^{a}$ Md. Humayun Kabir, ${ }^{b}$ Michitoshi Hayashi $^{c}$ and Keisuke Tominaga ${ }^{a b}$ \\ a Molecular Photoscience Research Center, Kobe University, Nada, Kobe, 657-8501, Japan \\ ${ }^{\boldsymbol{b}}$ CREST, JST, Nada, Kobe, 657-8501, Japan \\ ${ }^{c}$ Center for Condensed Matter Sciences, National Taiwan University, 1, Roosevelt Sec. 4, \\ Taipei, Taiwan, 106, R.O.C.
}

Received 27th January 2005, Accepted 10th March 2005

First published as an Advance Article on the web 1st April 2005

We have measured the frequency dependent extinction coefficients and refractive indices of electron donoracceptor (EDA) complexes consisting of hexamethylbenzene (HMB; electron donor) and tetracyanoethylene (TCNE; electron acceptor) in the low-frequency region by terahertz time-domain spectroscopy (THz-TDS). A mixture of the 1:1 (DA) and 2:1 ( $\left.\mathrm{D}_{2} \mathrm{~A}\right)$ EDA complexes exist in carbon tetrachloride solution, and we successfully obtained the spectral components of the $1: 1$ and $2: 1$ EDA complexes separately by analyzing the concentration dependence of the $\mathrm{THz}$ spectra. The $1: 1$ and $2: 1$ complexes show quite different $\mathrm{THz}$ spectra of the extinction coefficient, reflecting unique features of dynamics, fluctuations and intermolecular interactions of these complexes. Polarization-selective THz-TDS on the crystalline DA complex shows two peaks at 53 and 70 $\mathrm{cm}^{-1}$ in the spectral component perpendicular to the crystal axis. On the other hand, the crystalline $\mathrm{D}_{2} \mathrm{~A}$ complex exhibits peaks at 42 and $50 \mathrm{~cm}^{-1}$ in the perpendicular spectral component. We compare the obtained spectra of the crystalline complex and the results of molecular orbital calculations at the HF/6-31G(d) level of theory to discuss the intermolecular vibrational modes of the complexes.

\section{Introduction}

Vibrational spectroscopy has been widely used to investigate structures, interactions and dynamics of molecules and molecular complexes. The low-frequency region below several terahertz $\left(\mathrm{THz} ; 1 \mathrm{THz}=33.3 \mathrm{~cm}^{-1}\right)$ corresponds to intermolecular modes of complexes and intramolecular modes with a weaker potential force and/or larger reduced mass. ${ }^{1}$ Intermolecular interactions such as hydrogen bonding, van der Waals forces and charge-transfer interactions play important roles in various chemical and biological processes. ${ }^{2}$ Moreover, the lowfrequency spectra also reflect molecular dynamics on a time scale from picoseconds to femtoseconds. Electron donoracceptor (EDA) complexes are one of the typical non-covalently bonded systems, ${ }^{3}$ and their intermolecular interactions and dynamical fluctuations as well as electronic states and molecular structures have been studied for a couple of decades., ${ }^{2,3}$

EDA complexes consisting of hexamethylbenzene (HMB) and tetracyanoethylene (TCNE) have been extensively studied both experimentally and theoretically. ${ }^{2-25}$ One of the characteristic features of this system is its ability to form both $1: 1$ (DA) and 2:1 $\left(\mathrm{D}_{2} \mathrm{~A}\right)$ complexes. The charge-transfer absorption band in the visible region depends on the donor and acceptor concentrations, and thorough studies on the spectral changes as a function of the concentrations have determined association constants $K_{1}$ and $K_{2}$ for both the equilibration processes (eqn. (1)), ${ }^{4,6}$ $\dagger$ Current Address: Institute of Laser Engineering, Osaka University, 2-6 Yamadaoka, Suita, Osaka 565-0871, Japan.
Generally, the far-infrared (IR) region corresponds to intermolecular motions of the complexes, and microscopic information on the charge-transfer complexes such as intermolecular interactions and structural fluctuations can be studied by the far-IR spectra. There has been much effort to investigate the intermolecular vibrational band of the HMB-TCNE complex by optical absorption, ${ }^{4-6}$ IR absorption, ${ }^{7-11}$ resonance-Raman scattering, ${ }^{12-18}$ X-ray crystallography, ${ }^{19}$ time-resolved measurements, ${ }^{20,21}$ and molecular orbital calculation. ${ }^{22-25}$ However, it is still an open question to clarify the low-frequency vibrational mode of the complex. The $1: 1$ complex has a band at around $165 \mathrm{~cm}^{-1}$ in resonance-Raman spectra, ${ }^{12-18}$ and this band was assigned to the first overtone of the D-A stretching vibration. ${ }^{12}$ Rubtsov and Yoshihara performed femtosecond fluorescence up-conversion experiments on this complex in solution and assigned the band at around $165 \mathrm{~cm}^{-1}$ to an intramolecular vibrational mode of TCNE from the analysis of their fluorescence decay data. ${ }^{21}$ On the other hand, the complex in the solid state shows a band at $89 \mathrm{~cm}^{-1}$, which was assigned to an intermolecular vibration between HMB and TCNE. ${ }^{9}$ Hayashi et al. reported the normal mode calculations for the HMB-TCNE complex at HF/3-21G level theory and assigned the DA stretching mode at $59 \mathrm{~cm}^{-1} \cdot{ }^{23}$

In this work we have measured the low-frequency spectra of the HMB-TCNE complex in solution by $\mathrm{THz}$ time-domain spectroscopy (TDS) and obtained spectra of DA and $\mathrm{D}_{2} \mathrm{~A}$ in the $\mathrm{THz}$ region separately. We have also measured the $\mathrm{THz}$ spectra of the DA and $\mathrm{D}_{2} \mathrm{~A}$ complexes in the crystalline state by polarization-selective THz-TDS. We performed ab initio calculations on the DA and $\mathrm{D}_{2} \mathrm{~A}$ complexes at the $\mathrm{HF} /$ 6-31G(d) level to obtain harmonic vibrational frequencies of the low-frequency modes. The reorientational fluctuation of the DA complex in solution will be discussed. 


\section{Experimental and calculations}

The THz-TDS apparatus has been described in detail elsewhere. ${ }^{26}$ Briefly, femtosecond pulses from a mode-locked titanium sapphire laser are used for generation and detection of the pulsed $\mathrm{THz}$ radiation. A major part of the pulse is focused to a (100)-surface of an InAs wafer with an angle of incidence of $45^{\circ}$. The wafer is placed in a magnetic field with a flux density of $1.6 \mathrm{~T}$ to enhance the intensity of the radiation. The rest of the pulse is used to detect an electric field of the pulsed $\mathrm{THz}$ radiation by the electro-optic (EO) sampling method with a $\langle 110\rangle \mathrm{ZnTe}$ crystal (1 $\mathrm{mm}$ in thickness) as an EO crystal. The power spectrum of the $\mathrm{THz}$ radiation is shown in Fig. 1.

HMB, TCNE and carbon tetrachloride $\left(\mathrm{CCl}_{4}\right)$ were purchased from Wako Pure Chemicals and used without further purification. The concentrations of HMB and TCNE in solutions are listed in Table 1. The sample solutions were contained in a cell with an optical path length of $15 \mathrm{~mm}$. The cell windows are made of poly(tetrafluoroethylene) of $5 \mathrm{~mm}$ in thickness. Two identical cells were prepared, one of which is the reference and the other is for the sample solution. We measured $\mathrm{THz}$ temporal waveforms of the reference and sample solutions in succession. We also prepared crystalline samples of the $1: 1$ and $2: 1$ complexes by dissolving HMB and TCNE with $1: 1$ or $2: 1$ stoichiometrical ratios, respectively, in diethyl ether, and the solvent was evaporated slowly. ${ }^{10,19}$ Needle-shaped crystals were obtained for the $1: 1$ and $2: 1$ complexes. These were collected and aligned with each other to measure parallel and perpendicular components of the $\mathrm{THz}$ spectra. All the measurements were done at ambient temperature $\left(20^{\circ} \mathrm{C}\right)$.

In the analysis of the $\mathrm{THz}$ spectra of the solution we consider the difference between the reference and the sample cells by obtaining the amplitude variance $\eta(\tilde{\nu})$ and the phase variance $\xi(\tilde{\nu})$,

$$
\frac{E_{\mathrm{S}, \mathrm{CCl}_{4}}(\tilde{\nu})}{E_{\mathrm{R}, \mathrm{CCl}_{4}}(\tilde{\nu})}=\exp (\mathrm{i} \xi(\tilde{\nu})+\eta(\tilde{\nu}))
$$

$E_{\mathrm{R}, \mathrm{CCl}_{4}}(\tilde{\nu})$ and $E_{\mathrm{S}, \mathrm{CCl}_{4}}(\tilde{\nu})$ are the Fourier transformation of the $\mathrm{THz}$ waveforms transmitted through the reference cell and the sample cell, both filled with $\mathrm{CCl}_{4}$, respectively; $\tilde{\nu}$ denotes the wavenumber. The $\mathrm{THz}$ spectra are expressed as

$$
\begin{gathered}
\Delta n(\tilde{\nu})=\frac{1}{2 \pi \tilde{\nu} d}\left\{\arg \left(\frac{E_{\mathrm{S}}(\tilde{\nu})}{E_{\mathrm{R}}(\tilde{\nu})}\right)-\xi(\tilde{\nu})\right\} \\
\Delta \kappa(\tilde{\nu})=-\frac{1}{2 \pi \tilde{\nu} d}\left\{\ln \left(\left|\frac{E_{\mathrm{S}}(\tilde{\nu})}{E_{\mathrm{R}}(\tilde{\nu})}\right|\right)-\eta(\tilde{\nu})\right\}
\end{gathered}
$$

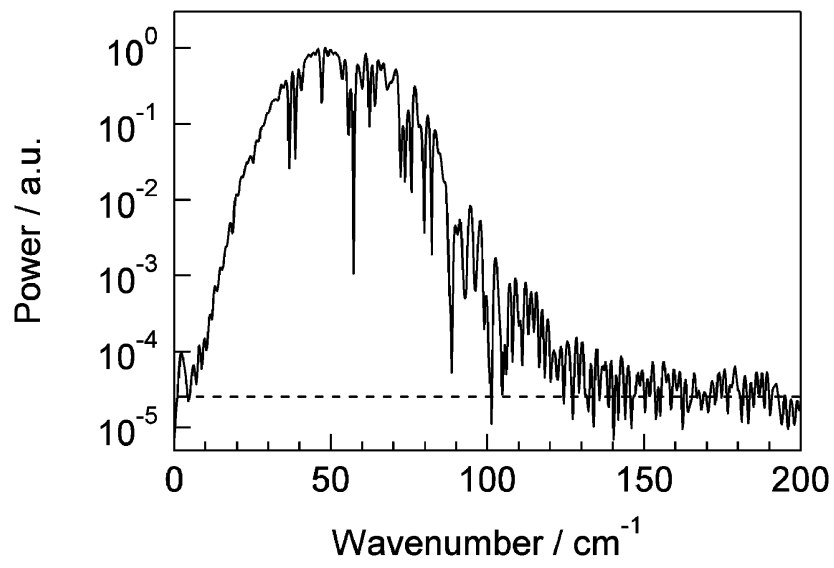

Fig. 1 A power spectrum of the THz radiation. Sharp spikes in the spectrum are due to absorption by water vapor in air. The broken line indicates the noise level of the system.
Table 1 Concentrations of the DA and DAD complexes in the carbon tetrachloride solutions

\begin{tabular}{lllll}
\hline$\left[\mathrm{D}_{0}\right]^{a} / \mathrm{mM}$ & {$\left[\mathrm{A}_{0}\right]^{a} / \mathrm{mM}$} & {$[\mathrm{DA}] / \mathrm{mM}$} & {$[\mathrm{DAD}] / \mathrm{mM}$} & {$\left[\mathrm{D}_{\text {unbound }}\right]^{b} / \mathrm{mM}$} \\
\hline 500 & 50 & 7.3 & 42.6 & 407.5 \\
350 & 30 & 5.7 & 24.2 & 295.9 \\
500 & 25 & 3.3 & 21.6 & 453.4 \\
250 & 20 & 4.9 & 15.0 & 215.1 \\
125 & 10 & 3.8 & 6.0 & 109.2 \\
40 & 6 & 3.7 & 1.7 & 32.9
\end{tabular}

${ }^{a}$ Initial concentrations. ${ }^{b}$ Concentration of HMB uncomplexed with TCNE.

$$
\begin{aligned}
\Delta \alpha(\tilde{\nu}) & =\frac{4 \pi \tilde{\nu}}{\ln 10} \Delta \kappa(\tilde{\nu}) \\
& =-\frac{1}{\ln 10} \frac{2}{d}\left\{\ln \left(\left|\frac{E_{\mathrm{S}}(\tilde{\nu})}{E_{\mathrm{R}}(\tilde{\nu})}\right|\right)-\eta(\tilde{\nu})\right\}
\end{aligned}
$$

where $d$ is the sample thickness; $\Delta n(\tilde{\nu}), \Delta \kappa(\tilde{\nu})$ and $\Delta \alpha(\tilde{\nu})$ are the difference spectra of refractive index, extinction coefficient and absorption coefficient, respectively.

We have calculated the ground-state geometries, normal modes of vibration, dipole moments and interaction energies of the DA and $\mathrm{D}_{2} \mathrm{~A}$ complexes of HMB with TCNE using the Hartree-Fock (HF) theory and a medium-sized basis set. The Gaussian 03 package $^{27}$ was used for the HF calculations with the $6-31 G(d)$ basis set. Full geometry optimizations were performed for the monomers and the complexes without imposing any constraints, and the resultant geometries were
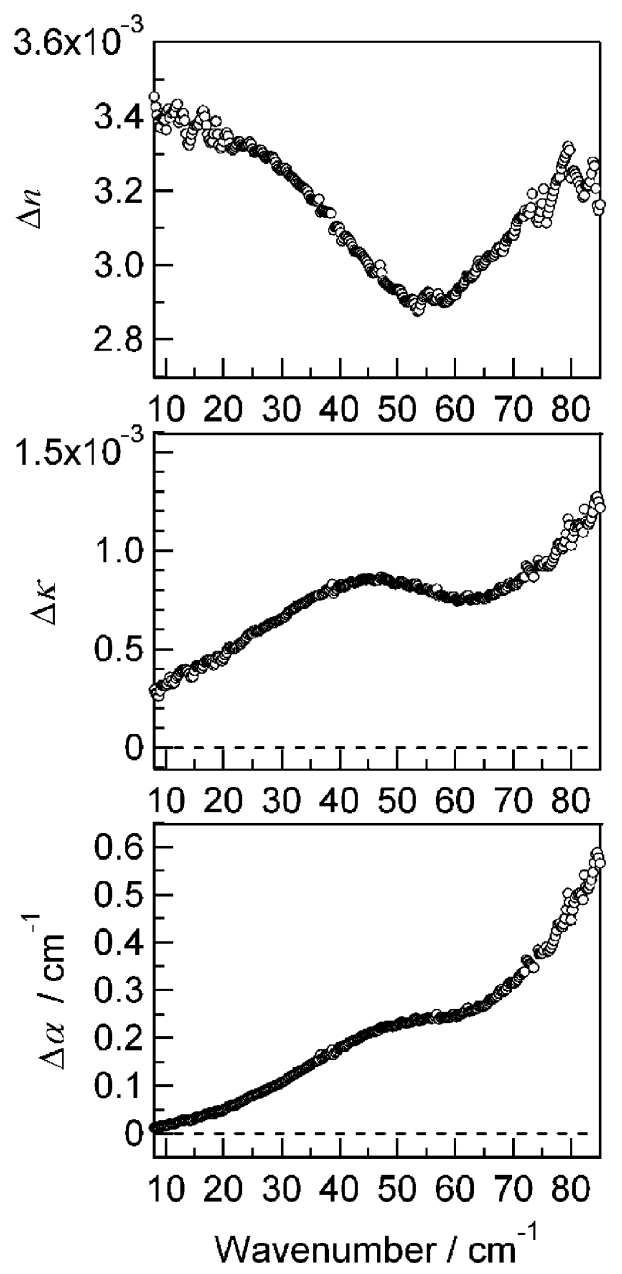

Fig. 2 Difference spectra between $\mathrm{HMB} / \mathrm{TCNE}$ in $\mathrm{CCl}_{4}(500 \mathrm{mM} / 50$ $\mathrm{mM})$ and $\mathrm{HMB}$ in $\mathrm{CCl}_{4}(500 \mathrm{mM})$. (Top) Refractive index, (middle) extinction coefficient and (bottom) absorption coefficient. 
confirmed through the frequency calculations to represent the minima on the respective potential energy surface. Binding energy $\left(\Delta E_{\mathrm{e}}\right)$ was corrected for basis set superposition error (BSSE) by means of the counterpoise correction of Boys and Bernardi. ${ }^{28}$

\section{Results}

\subsection{EDA complexes in solution}

Fig. 2 displays difference spectra of refractive index $(\Delta n(\tilde{\nu}))$, extinction coefficient $(\Delta \kappa(\tilde{\nu}))$ and absorption coefficient $(\Delta \alpha(\tilde{\nu}))$ between a solution of $\mathrm{HMB} / \mathrm{TCNE}$ in $\mathrm{CCl}_{4}(500 \mathrm{mM} / 50 \mathrm{mM})$ as a sample and a solution of $\mathrm{HMB}$ in $\mathrm{CCl}_{4}(500 \mathrm{mM})$ as a reference. An increase of the absorption was observed when TCNE was added into the HMB solution. This increase is due to the formation of the EDA complex. We measured concentration dependences of difference spectra of the refractive index and extinction coefficient or absorption coefficient of this system (Fig. 3). The reference for the measurement is a solution of $\mathrm{HMB}$ in $\mathrm{CCl}_{4}$ with the same concentration as that in the sample solution. In all the solutions an excess amount of HMB was added in comparison to TCNE, therefore, almost all the TCNE molecules form the complexes. In the frequency regions below $15 \mathrm{~cm}^{-1}$ and above $85 \mathrm{~cm}^{-1}$ the signal-to-noise ratio becomes worse, and we do not discuss results in these regions.

It can be clearly seen in Fig. 3 that both the refractive index and the extinction coefficient change their spectral shapes as well as the intensities as a function of the concentration. This indicates that the $\mathrm{DA}$ and $\mathrm{D}_{2} \mathrm{~A}$ complexes have different $\mathrm{THz}$ spectra from each other. We calculated frequency dependent spectra of refractive indices and extinction coefficients for DA and $\mathrm{D}_{2} \mathrm{~A}$, so that the concentration dependences of the ob- served spectra can be reproduced well by using concentrations of the DA and $\mathrm{D}_{2} \mathrm{~A}$ complexes estimated by the reported association constants for DA and $\mathrm{D}_{2} \mathrm{~A}\left(K_{1}=190.1 \pm 4.6 \mathrm{M}^{-1}\right.$ and $K_{2}=14.24 \pm 0.62 \mathrm{M}^{-1}$ at $\left.20{ }^{\circ} \mathrm{C}\right) .{ }^{6}$ The contribution of unbound $\mathrm{HMB}$ to the observed spectra was also considered. The concentrations of DA, $\mathrm{D}_{2} \mathrm{~A}$ and unbound HMB in the solutions investigated are listed in Table 1. The results are shown in Fig. 4. The concentration dependences of the refractive index and extinction coefficient in solution are reproduced well by using the obtained DA and $\mathrm{D}_{2} \mathrm{~A}$ spectra as shown by the solid lines in Fig. 3. The striking feature of the results is that $\mathrm{DA}$ and $\mathrm{D}_{2} \mathrm{~A}$ show very different spectra in the low-frequency region. The $\mathrm{D}_{2} \mathrm{~A}$ spectrum shows a broad band centered at $47 \mathrm{~cm}^{-1}$. DA has a larger molar extinction coefficient than $\mathrm{D}_{2} \mathrm{~A}$ in the frequency investigated. Moreover, the extinction coefficient in the very lowfrequency region $\left(<40 \mathrm{~cm}^{-1}\right)$ shows opposite dependence on the frequency with each other; in the case of DA, the spectral intensity decreases as a function of the frequency, while $\mathrm{D}_{2} \mathrm{~A}$ shows an increase with increasing the frequency.

\subsection{EDA complexes in the crystalline state}

The frequency-dependent extinction coefficients of the DA complex in the crystalline state are shown in Fig. 5. The needle-shaped crystals are aligned with each other, and the parallel and perpendicular components of the extinction coefficient with respect to the crystal axis were measured. The parallel component in the frequency range over $60 \mathrm{~cm}^{-1}$ could not be measured due to its strong absorption. The extinction coefficient of the parallel component is much larger than that of the perpendicular component. On the other hand, the perpendicular component shows peaks at around 53 and 70 $\mathrm{cm}^{-1}$. Both the parallel and perpendicular components (a)
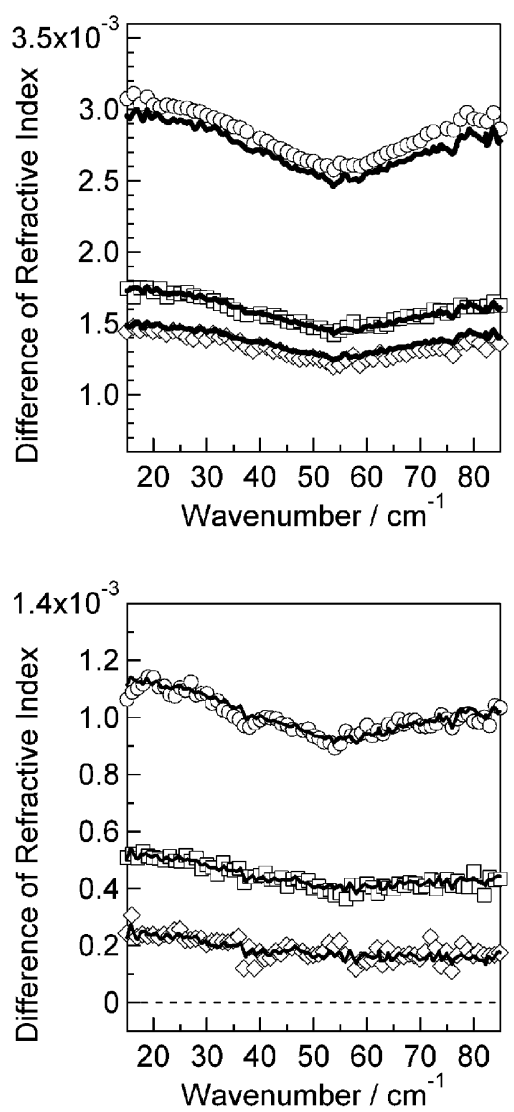

(b)
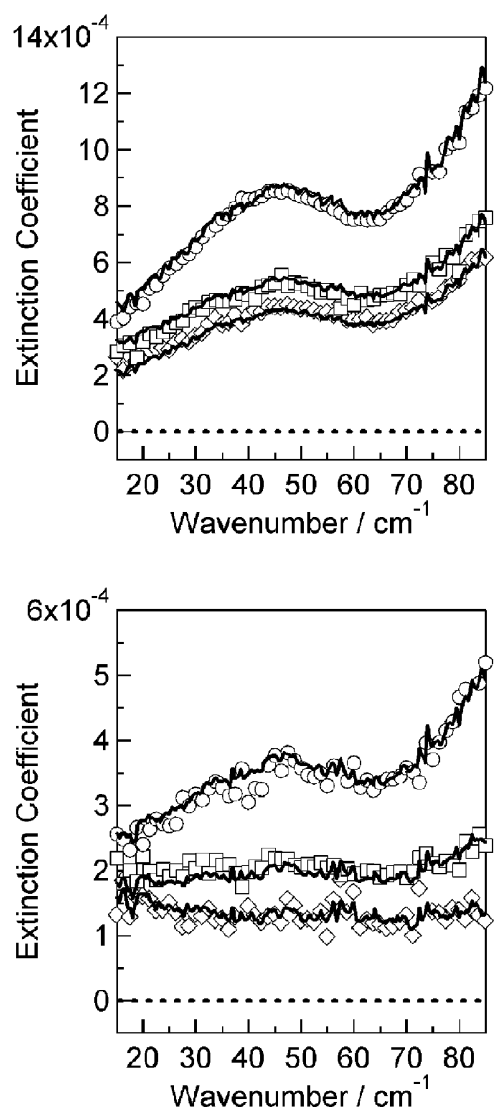

Fig. 3 (a) Refractive index changes (left) and extinction coefficients (right) of hexamethylbenzene (HMB) and tetracyanoethylene (TCNE) in carbon tetrachloride. The concentrations of HMB and TCNE are as follows from the top to bottom; ([HMB], [TCNE]) $=(500 \mathrm{mM}, 50 \mathrm{mM})$, $(350 \mathrm{mM}, 30 \mathrm{mM})$ and $(500 \mathrm{mM}, 25 \mathrm{mM})$. (b) Same as (a): ([HMB], [TCNE] $)=(250 \mathrm{mM}, 20 \mathrm{mM}),(125 \mathrm{mM}, 10 \mathrm{mM})$ and $(40 \mathrm{mM}, 6 \mathrm{mM})$. The solid lines are spectra reconstructed by using the results in Fig. 4. 

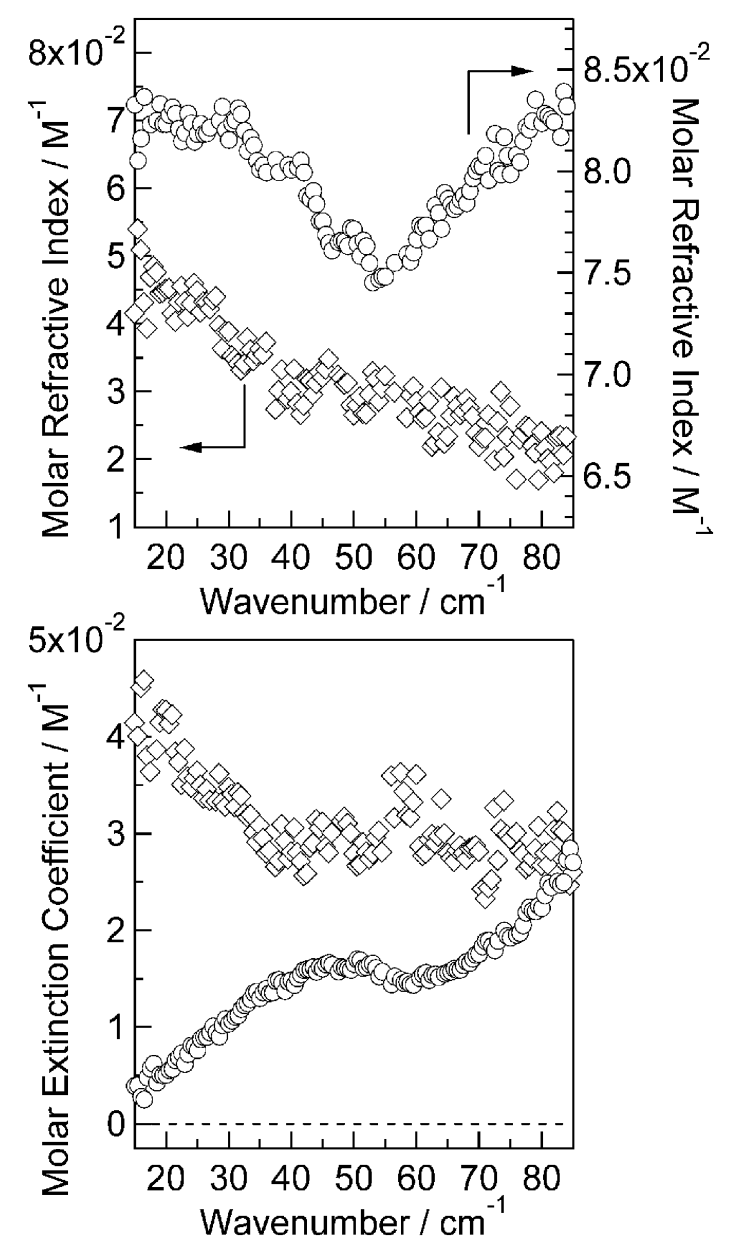

Fig. 4 Refractive indices (upper) and extinction coefficients (lower) per molar concentration of the DA $(\diamond)$ and DAD $(\bigcirc)$ complexes.

increase monotonically as a function of the frequency in the very low-frequency region below $40 \mathrm{~cm}^{-1}$. This spectral feature is in a sharp contrast to that in the solution phase. As shown in Fig. 6, the polarization-selective extinction coefficient spectra of the $\mathrm{D}_{2} \mathrm{~A}$ complex show similar features to the DA complex. The perpendicular component shows a peak at $50 \mathrm{~cm}^{-1}$ and a shoulder at $42 \mathrm{~cm}^{-1}$, whereas the parallel component exhibits a monotonic increase as a function of the frequency.

\subsection{Ab initio calculation}

The calculated structure of the HMB-TCNE complex with the minimum energy obtained at the $\mathrm{HF} / 6-31 \mathrm{G}(\mathrm{d})$ level is shown in

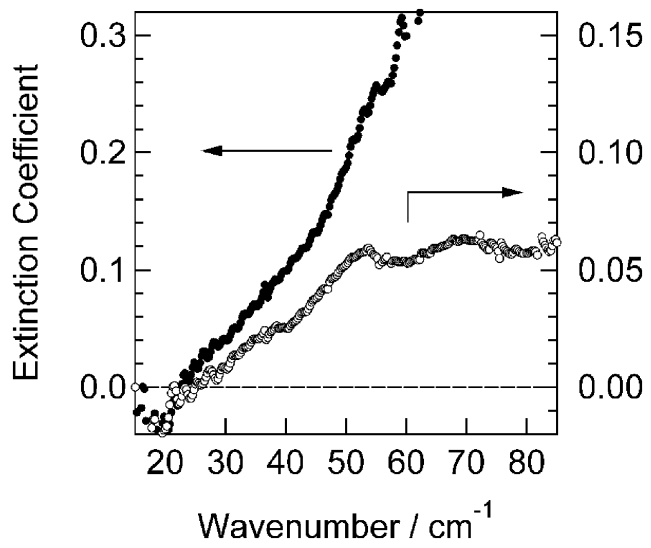

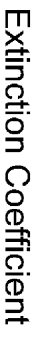

Fig. 5 The parallel $(O)$ and perpendicular $(\bigcirc)$ components of the frequency-dependent extinction coefficient of the DA complex in the crystalline state.

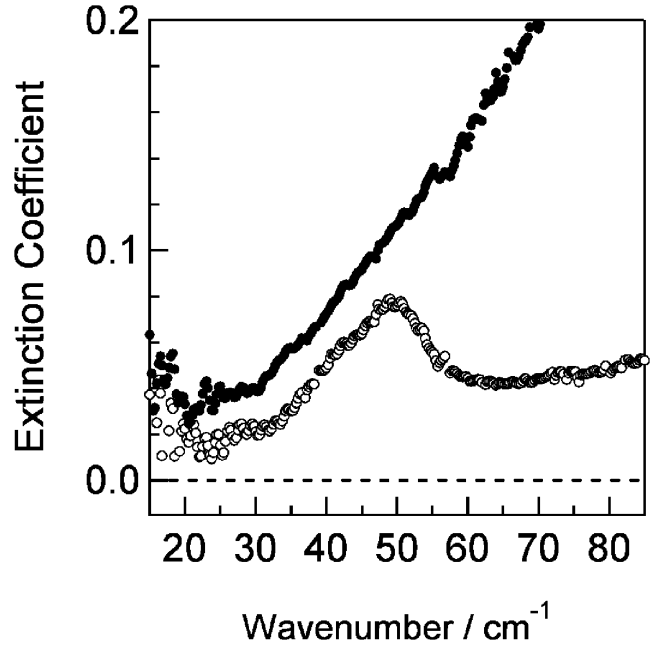

Fig. 6 The parallel $(O)$ and perpendicular $(O)$ components of the frequency-dependent extinction coefficient of the DAD complex in the crystalline state.

Fig. 7. The molecular planes are nearly parallel to each other (the dihedral angle between the planes of HMB and TCNE is $3^{\circ}$ ) and separated by $3.70 \AA$. The TCNE molecule is oriented with respect to the $\mathrm{HMB}$ molecule at an angle of $27.0^{\circ}$. The calculated structure agreed fairly well with the crystal structure. ${ }^{19}$ In accordance with the crystal structure, the interplanar dihedral angle and separation are $0.4^{\circ}$ and $3.33 \AA$, respectively, and the orientation angle of the TCNE molecule with respect to the $\mathrm{HMB}$ molecule is $9.0^{\circ}$. Harmonic vibrational frequencies were computed in vacuo for the monomers and the complex at the HF/6-31G(d) level. The donor-acceptor vibrational modes whose frequencies below $100 \mathrm{~cm}^{-1}$ are listed in Table 2 together with their assignments. Hayashi et al. also reported the normal mode calculations for the HMB-TCNE complex at $\mathrm{HF} / 3-21 \mathrm{G}$ level theory. ${ }^{23}$ They have found seven intermolecular modes at frequencies below $100 \mathrm{~cm}^{-1}$, two of which are highly mixed with $\mathrm{HMB} \mathrm{CH}_{3}$ twisting modes. Among these intermolecular modes they assigned one DA stretching mode at $59 \mathrm{~cm}^{-1}$. Fig. 8 illustrates some strongly IR active DA intermonomer modes predicted in our calculation. The binding energy of the DA complex was computed at the HF/6$31 \mathrm{G}(\mathrm{d})$ level without and with basis set superposition error (BSSE) correction by the counterpoise method. The equilibrium binding energy $\Delta E_{\mathrm{e}}$ was found to be -4.46 and $-2.83 \mathrm{kcal}$ $\mathrm{mol}^{-1}$ for the BSSE uncorrected and BSSE corrected structures, respectively. The enthalpy of complexation $\Delta H^{0}$ for the HMB-TCNE complex in solution has been reported to be $-7.9 \mathrm{kcal} \mathrm{mol}^{-1}$ in $\mathrm{CCl}_{4}{ }^{6}$. The dipole moment for the DA complex calculated at $\mathrm{HF} / 6-31 \mathrm{G}(\mathrm{d})$ level is $0.92 \mathrm{D}$, which is smaller than the experimental value of $2.8 \mathrm{D}^{4}$ Since energies, intermolecular distances, and other properties of the complexes

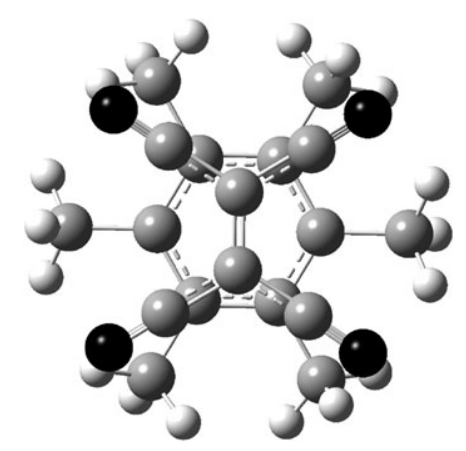

Fig. 7 The calculated structure of the HMB-TCNE complex with minimum energy obtained by $a b$ initio calculation at the $\mathrm{HF} / 6-31 \mathrm{G}(\mathrm{d})$ level. 
Table 2 Vibrational modes of the HMB-TCNE complex below $100 \mathrm{~cm}^{-1}$ calculated at $\mathrm{HF} / 6-31 \mathrm{G}(\mathrm{d})$ level

\begin{tabular}{rlll}
\hline Vibrational frequency $/ \mathrm{cm}^{-1}$ & IR intensity $/ \mathrm{km} \mathrm{mol}^{-1}$ & Force constant $/ \mathrm{mDyn}^{-1}$ & Approximate vibrational mode assignment \\
\hline 2.41 & 0.0009 & $<0.0001$ & HMB-TCNE twisting \\
31.86 & 0.1339 & 0.0052 & HMB-TCNE sliding \\
34.29 & 0.1936 & 0.0042 & HMB-TCNE sliding \\
49.90 & 0.0119 & HMB-TCNE stretching \\
53.80 & 0.0041 & 0.0098 & HMB-TCNE tilting \\
64.63 & 0.0147 & 0.011 & HMB-TCNE tilting \\
87.48 & 0.0007 & 0.0056 & HMB CH $\mathrm{H}_{3}$ twisting and TCNE C-C $\equiv \mathrm{N}$ out-of-plane torsion \\
94.36 & 0.0034 & 0.0125 & HMB CH $\mathrm{H}_{3}$ twisting and TCNE C-C $\equiv$ out-of-plane torsion \\
97.67 & 0.0815 & 0.0084 & \\
\hline
\end{tabular}

are markedly dependent on the theoretical model and the level of the theory, higher level calculations are on going in our laboratory and the results will be published elsewhere. ${ }^{29}$

The calculated minimum energy structure of the HMBTCNE-HMB complex obtained at the HF/6-31G(d) level is shown in Fig. 9. The molecular planes are nearly parallel to each other and separated by $3.77 \AA$. The TCNE molecule oriented with respect to the HMB molecules at an angle $12.9^{\circ}$ Harmonic vibrational frequencies were computed in vacuo for the complex at the $\mathrm{HF} / 6-31 \mathrm{G}(\mathrm{d})$ level. The donor-acceptordonor vibrational modes whose frequencies are below $70 \mathrm{~cm}^{-1}$

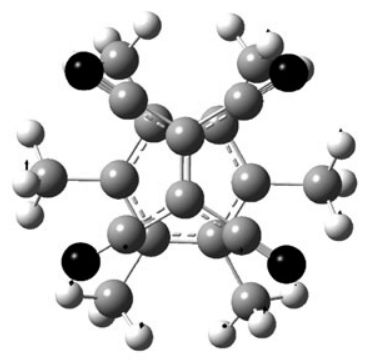

(a)
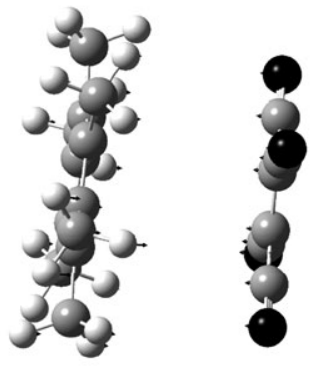

(c)
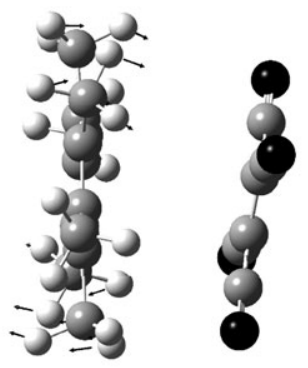

(e)

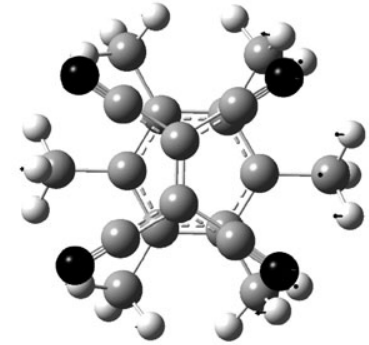

(b)

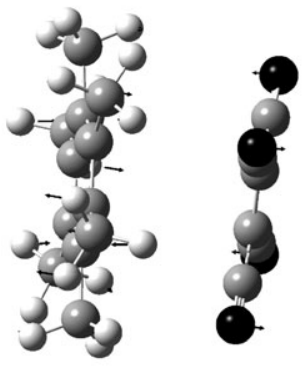

(d) are listed in Table 3 with their assignments. Fig. 10 illustrates some of the IR active modes predicted in our calculation. The binding energy of the $\mathrm{D}_{2} \mathrm{~A}$ complex was computed at the $\mathrm{HF} / 6$ $31 G(d)$ level without basis set superposition error correction. The equilibrium binding energy $\Delta E_{\mathrm{e}}$ was found to be -8.53 $\mathrm{kcal} \mathrm{mol}{ }^{-1}$. The dipole moment for the $\mathrm{D}_{2} \mathrm{~A}$ complex calculated at $\mathrm{HF} / 6-31 \mathrm{G}(\mathrm{d})$ level is $0.04 \mathrm{D}$, which is close to the experimental value of $0.0 \mathrm{D},{ }^{4}$ suggesting that the structure of the $2: 1$ complex is DAD.

\section{Discussion}

In this section we first compare the experimental results for the crystalline states with the theoretical calculations. Although the basis set we have used is a higher one than previously ${ }^{23}$ and the level of the calculation has been improved, the present theoretical method can not still properly treat the intermolecular interactions between the donor and acceptor. We need further improvement by including effects of, for example, electron correlation or polarization dispersion to discuss the vibrational assignment in a quantitative level. Furthermore, the calculation was done for the isolated case in the vacuum, whereas the experimental data were taken in the crystalline state. Nevertheless, it can still give a guideline for discussing the vibrational assignment in a qualitative level such as ordering of the vibrational modes as a function of the frequency or relative intensities of the vibrational bands.

First let us discuss the intermolecular vibrational modes of the DA complex. The extinction coefficient spectrum does not show any sharp band in solution, and neither does the parallel component of the crystalline-state spectrum. However, the perpendicular component of the crystalline state shows peaks at 53 and $70 \mathrm{~cm}^{-1}$. The $\mathrm{HF} / 6-31 \mathrm{G}(\mathrm{d})$ calculation predicts three bands at $31.86,34.29$ and $49.90 \mathrm{~cm}^{-1}$ which have reasonable
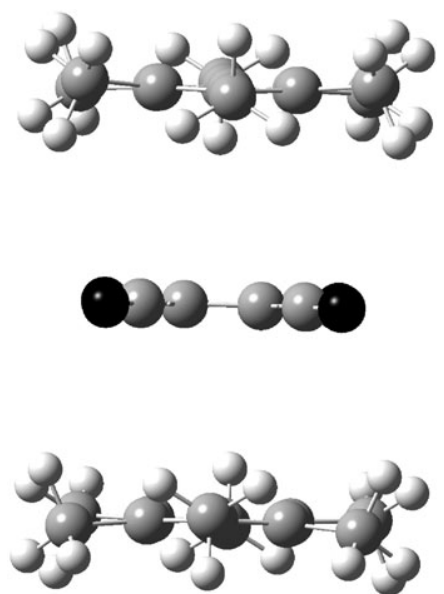

Fig. 8 Strongly IR active modes of the DA complex below $100 \mathrm{~cm}^{-1}$ obtained by ab initio calculation at the $\mathrm{HF} / 6-31 \mathrm{G}(\mathrm{d})$ level. The frequencies are 31.86 (a), 34.29 (b), 49.90 (c), 53.80 (d) and 64.63 $\mathrm{cm}^{-1}$ (e). The arrows indicate the displacement vectors corresponding to these low-frequency modes. 
Table 3 Vibrational modes of the HMB-TCNE-HMB complex below $70 \mathrm{~cm}^{-1}$ calculated at $\mathrm{HF} / 6-31 \mathrm{G}(\mathrm{d})$ level

\begin{tabular}{lccl}
\hline Vibrational frequency $/ \mathrm{cm}^{-1}$ & IR Intensity $/ \mathrm{km} \mathrm{mol}^{-1}$ & Force constant $/ \mathrm{mDyn}^{-1}$ & Approximate vibrational mode assignment \\
\hline 1.51 & $<0.0001$ & $<0.0001$ & D-A-D twisting \\
4.71 & 0.006 & 0.0001 & D-A-D twisting \\
18.41 & 0.1741 & 0.0014 & D-A-D sliding \\
22.19 & 0.2304 & 0.0020 & D-A-D sliding \\
30.55 & 0.0067 & 0.0024 & D-A-D sliding \\
31.21 & 0.0048 & 0.0028 & D-A-D sliding \\
32.86 & 0.0004 & 0.0035 & D-A-D stretching \\
42.52 & 0.19 & 0.0042 & D-A-D sliding \\
56.69 & 0.19 & 0.0074 & D-A-D sliding \\
60.19 & 2.25 & 0.0197 & D-A-D stretching \\
67.64 & 0.005 & 0.0207 & D-A-D tilting \\
72.92 & 0.0006 & 0.0173 & D-A-D tilting \\
\hline
\end{tabular}

IR intensities. The first two modes are assigned as D-A sliding modes and the last one is assigned as a $\mathrm{D}-\mathrm{A}$ stretching mode. Currently, we are performing molecular orbital calculations with different methods with various basis sets on the same system, and the preliminary results show that the frequencies of these bands obtained by the density functional theory are about one and half times larger than the present values. ${ }^{29}$ For the DA case, we tentatively assign the $53 \mathrm{~cm}^{-1}$ band observed in the perpendicular component as a DA sliding mode, because the transition dipole moment is oriented perpendicular to the crystal axis. The perpendicular component also shows a broad peak at around $70 \mathrm{~cm}^{-1}$. The calculation predicts a DA stretching mode with a resonance frequency at $49.90 \mathrm{~cm}^{-1}$. According to the calculation, this band has the

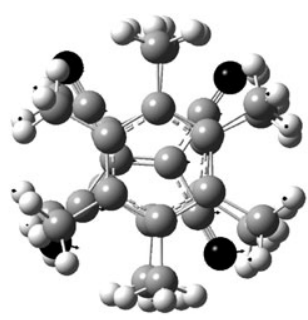

(a)
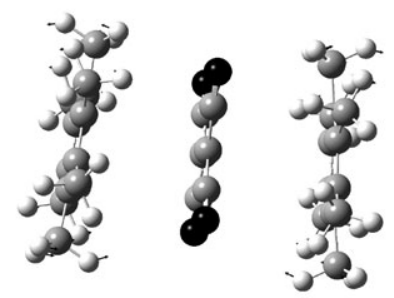

(c)

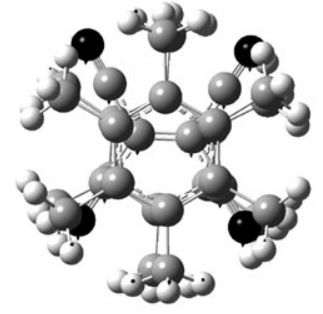

(b)

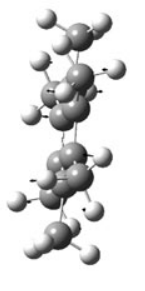

(d)
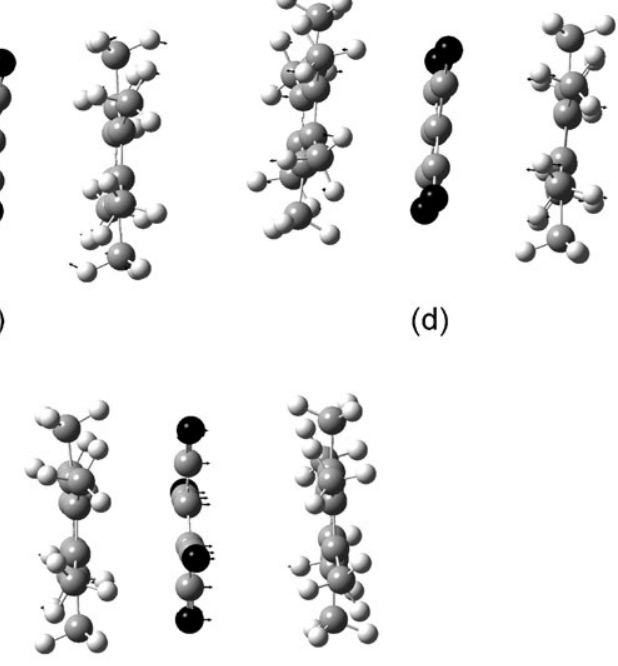

(e)

Fig. 10 Strongly IR active modes of the DAD complex below $70 \mathrm{~cm}^{-1}$ obtained by ab initio calculation at the $\mathrm{HF} / 6-31 \mathrm{G}(\mathrm{d})$ level. The frequencies are 18.41 (a), 22.19 (b), 42.52 (c), 56.69 (d) and 60.19 $\mathrm{cm}^{-1}$ (e). The arrows indicate the displacement vectors corresponding to these low-frequency modes. strongest IR activity among the modes below $100 \mathrm{~cm}^{-1}$. Although the direction of the transition dipole moment of this mode is considered to be parallel with the crystal axis, the prepared sample may have a distribution of the direction of the crystal axis. These possibilities may give a spectral component even in the perpendicular component, suggesting that the $70 \mathrm{~cm}^{-1}$ band in the perpendicular component may be due to the DA stretching mode.

For the $\mathrm{D}_{2} \mathrm{~A}$ complex, the spectrum in solution shows a broad peak at $47 \mathrm{~cm}^{-1}$, and the perpendicular component of spectrum in the crystalline state exhibits bands at 42 and 50 $\mathrm{cm}^{-1}$, though there is no distinct band in the parallel component. The $\mathrm{HF} / 6-31 \mathrm{G}(\mathrm{d})$ calculation shows that there are five bands with reasonable sizes of the IR intensity at 18.41, 22.19, $42.52,56.69$ and $60.19 \mathrm{~cm}^{-1}$. The first four modes are assigned as DAD sliding modes and the last one is assigned as a DAD stretching mode. We tentatively assign the bands at 42 and $50 \mathrm{~cm}^{-1}$ as DAD sliding modes, because if they are due to the stretching motion, it is expected that the parallel component should show peaks at these frequencies. However, there is no observation of such peaks in the parallel component. The distinct peak in the perpendicular component indicates the transition dipole of this band is oriented perpendicular to the crystal axis. Similarly, it is suggested that the band at $47 \mathrm{~cm}^{-1}$ observed in solution may be also a DAD sliding mode, though the frequency is different from those observed in the crystalline state. The difference may be due to different structures in solution caused by interaction with surrounding solvents.

It should be also noted that a vibrational band in the far-IR region often shows temperature dependence. ${ }^{30}$ Normally the vibrational peak shifts to a higher frequency as the temperature is lowered. This is partly because there are several vibrational transitions from higher energy levels which are thermally populated in the case of the far-IR spectrum. The results of the ab initio calculation should be, in principle, compared with a spectrum measured at low temperatures.

The far-IR spectrum corresponds to Fourier transformation of the time-correlation function (TCF) of the total dipole moment of the system, $\mathbf{M}(t)$, expressed as, ${ }^{31}$

$$
\begin{aligned}
\alpha(\omega)= & \frac{2 \pi}{3 \hbar c n(\omega)} \omega\left(1-\exp \left(-\hbar \omega / k_{\mathrm{B}} T\right)\right) \\
& \times \int_{-\infty}^{+\infty} \mathrm{d} t \exp (-\mathrm{i} \omega t)\langle\mathbf{M}(t) \cdot \mathbf{M}(0)\rangle
\end{aligned}
$$

where $c$ is the speed of light, $\omega$ is the angular frequency and $k_{\mathrm{B}} T$ has its usual meaning. Two types of molecular motions in condensed phases contribute to the far-IR spectrum; one is the reorientational relaxation of a permanent and/or induced dipole of the molecule, and the other is inter- or intramolecular vibrational motion. If the molecule or the complex in solution does not possess a permanent dipole moment, the 
reorientational relaxation contribution should be minor unless an induced dipole moment is significant. In solution we have found that the spectral feature differs in the very low-frequency region below $40 \mathrm{~cm}^{-1}$ between the $\mathrm{DA}$ and $\mathrm{D}_{2} \mathrm{~A}$ complexes. This is related to the difference of the dipole moment in the electronic ground state. The dipole moment of DA was estimated to be $2.8 \mathrm{D}$ by optical absorbance measurement and dielectric constant data. ${ }^{4}$ On the other hand, that of $\mathrm{D}_{2} \mathrm{~A}$ was reported to be $0 \mathrm{D}$, suggesting that the $\mathrm{D}_{2} \mathrm{~A}$ complex has a $D_{2 \mathrm{~h}}$ symmetry. ${ }^{4,6}$ Therefore, orientational relaxation does not contribute to the $\mathrm{THz}$ spectra of $\mathrm{D}_{2} \mathrm{~A}$ significantly. The DA complex shows a decrease of the extinction coefficient in the very low-frequency region as a function of the frequency. Considering the fact that the DA complex has a permanent dipole moment, this suggests that the spectral component in the very low-frequency region results from the reorientational relaxation of the dipole moment. In the extremely low-frequency region the $a b$ initio calculation predicts a vibrational band at $2.41 \mathrm{~cm}^{-1}$. The IR activity of this band is found to be negligibly weak compared to that of the DA stretching band at $49.90 \mathrm{~cm}^{-1}$, suggesting that the very low-frequency component of DA is purely due to the reorientational relaxation. The crystalline sample shows a monotonic increase as a function of the wavenumber in the very low-frequency region. This also supports the idea that the spectral component of below $40 \mathrm{~cm}^{-1}$ originates from the reorientational dynamics of the dipole of DA. The intermolecular vibrational modes may be inhomogeneously broadened in solution, and the anharmonicity makes the spectrum broader for a mode whose frequency is much lower than that of the thermal energy at room temperature. Furthermore, anharmonicity makes a vibration more susceptible to the frequency fluctuations caused by the surrounding solvent molecules. ${ }^{32,33}$ These may be the reason why the DA complex in solution does not show any structure in the spectrum above $40 \mathrm{~cm}^{-1}$.

To allow a more quantitative discussion, we simulated the $\mathrm{THz}$ spectrum of the DA complex in terms of eqn. (3) by focusing on the very low-frequency region from 15 to $40 \mathrm{~cm}^{-1}$. First, the experimental results are recast into the power spectrum of the TCF of $\mathbf{M}(t),\langle\mathbf{M}(t) \cdot \mathbf{M}(0)\rangle$, by Fourier transformation (Fig. 11). We assumed that the TCF of the total dipole moment is a sum of the TCF of the individual dipole moment $\langle\mu(t) \cdot \mu(0)\rangle,\langle\mathbf{M}(t) \cdot \mathbf{M}(0)\rangle=N\langle\mu(t) \cdot \mu(0)\rangle$, where $N$ is the number of the dipoles. Both exponential and gaussian functions are used as a model function for the TCF to simulate the power spectra. The exponential function provides a better simulation result. It was found that the time constant for both the functions should be in the range of sub-picoseconds. However, it is unlikely that such a weakly binding complex physically rotates in a rigid manner with a time constant

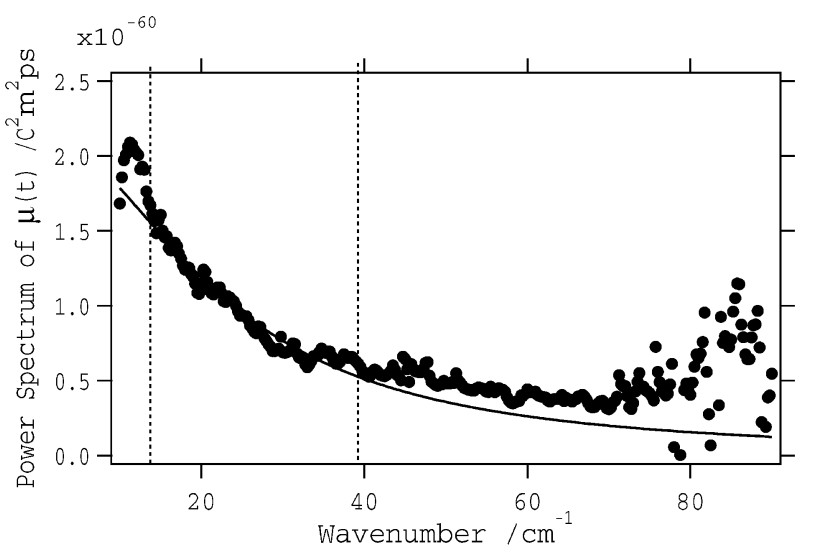

Fig. 11 A power spectrum obtained by Fourier transformation of the time correlation function of the individual dipole moment of the DA complex. The solid line is the result of the simulation by eqn. (4) in the region from 15 to $40 \mathrm{~cm}^{-1}$. See text for details. shorter than a picosecond. We propose the following model to explain the very low-frequency spectrum of the DA complex; surrounding solvent molecules collide with the complex continuously in solution, causing fluctuations of the direction and the size of the dipole of the DA complex. Since the collision frequency in solution is typically a few hundreds of femtoseconds, it is plausible that the very low frequency component of the frequency-dependent extinction coefficient of the DA complex results from collision-induced fluctuation of the dipole. Another interpretation for such a fast orientational relaxation time may be that the lifetime of the complex dominates the decay of the TCF of the dipole moment. However, if the lifetime of the complex is in the sub-picosecond time scale, the resonance-Raman bands of the complex are expected to show lifetime broadening with more than $10 \mathrm{~cm}^{-1}$, which was not experimentally observed. ${ }^{17}$

In order to examine the proposed model, we used the following model function for the simulation. The size of the dipole moment of the complex $\mu(t)$ oscillates with its resonance frequency, $\mu(t)=\Delta \mu(t)+\mu_{0}(t)$, where $\Delta \mu(t)$ describes an oscillating component of the dipole and $\mu_{0}(t)$ is the average dipole moment. $\mu_{0}(t)$ does not change its size in time, but the direction of the moment varies. The TCF consists of three parts, TCFs of $\Delta \mu(t)$ and $\mu_{0}(t)$ and its cross term. The TCF of $\Delta \mu(t)$ gives vibrational aspects in the spectrum, which is not observed in the solution phase. If we assume that there is no correlation between the oscillation and the reorientational relaxation, the cross term can be neglected. Therefore, we assumed $\langle\mu(t) \cdot \mu(0)\rangle=\left\langle\mu_{0}(t) \cdot \mu_{0}(0)\right\rangle$. Next, the TCF is divided into two parts, one is a fast relaxation component giving a spectrum in the $\mathrm{THz}$ spectrum and a slow relaxation component which corresponds to the orientational relaxation described by a hydrodynamic theory such as the Stokes-Einstein-Debye model,

$$
\begin{aligned}
\langle\mu(t) \cdot \mu(0)\rangle= & a_{\text {fast }} \mu^{2} \exp \left(-t / \tau_{\text {fast }}\right) \\
& +a_{\text {slow }} \mu^{2} \exp \left(-t / \tau_{\text {slow }}\right)
\end{aligned}
$$

Here $\mu$ is the dipole moment of the DA complex of $2.8 \mathrm{D}$, and a sum of two coefficients $a_{\text {fast }}$ and $a_{\text {slow }}$ is unity. The slow component contributes to the spectrum only around $0 \mathrm{~cm}^{-1}$, and in the simulation we neglected the second term. We simulated the spectrum from 15 to $40 \mathrm{~cm}^{-1}$, and the result is shown in Fig. 11. From the simulation we obtained $a_{\text {fast }}=0.10$ and $\tau_{\text {fast }}=$ 0.24 ps. The error bars for these values are about $\pm 10 \%$. The results suggest that $10 \%$ of the dipole fluctuation occurs in a subpicosecond time scale, which may be due to collision with the solvent molecules. This results show that spectra in the $\mathrm{THz}$ region contains information on the fluctuation of the dipoles with a sub-picosecond time scale in solution, and not only the time constant of the fluctuation, but also the magnitude of the fluctuations are estimated by the spectral simulation.

\section{Summary}

In conclusion, we have performed spectroscopic measurements in the far-IR region on the electron donor-acceptor complexes of hexamethylbenzene and tetracyanoethylene in solution and in the crystalline state by THz-TDS. We have also performed molecular orbital calculations using the Hartree-Fock theory with the $6-31 \mathrm{G}(\mathrm{d})$ basis set. The perpendicular component of the extinction coefficient of the crystalline DA complex shows peaks at 53 and $70 \mathrm{~cm}^{-1}$, which the molecular orbital calculation suggests originates from the DA sliding and DA stretching modes, respectively. The crystalline $\mathrm{D}_{2} \mathrm{~A}$ complex exhibits peaks at 42 and $53 \mathrm{~cm}^{-1}$ in the perpendicular component, and we tentatively assigned these modes as the DAD sliding modes. The frequency dependent extinction coefficients markedly differ between the DA and $\mathrm{D}_{2} \mathrm{~A}$ complexes in $\mathrm{CCl}_{4}$ solution. The difference mainly arises from the difference of the dipole moments of the two complexes. We have observed a 
behavior of the dipole reorientational relaxation in the frequency dependent extinction coefficient of DA in solution. The time constant of this relaxation is found to be in a range of subpicoseconds, which may be due to dipole fluctuations induced by collision with solvent molecules.

\section{Acknowledgements}

This work was supported by a Grant-In-Aid (10206101) from the Ministry of Education, Science, Sports and Culture, and a JSPS research grant for the Future Program.

\section{References}

1 K. D. Möller and W. G. Rothschild, Far-infrared Spectroscopy, Wiley, New York, 1971

2 Spectroscopy and Structure of Molecular Complexes, ed. J. Yarwood, Plenum Press, London, 1973.

3 (a) R. S. Mulliken, J. Am. Chem. Soc., 1950, 72, 600-608; (b) R. S Mulliken, J. Am. Chem. Soc., 1952, 74, 811-824; (c) R. S. Mulliken, J. Phys. Chem., 1952, 56, 801-822.

4 R. Forster and N. Kulevsky, J. Chem. Soc., Faraday Trans. 1, 1973, 69, 1427-1431.

5 M. Rossi, U. Buser and E. Haselbach, Helv. Chim. Acta, 1976, 59, 1039-1053.

6 W. Liptay, T. Rehm, D. Wehning, L. Schanne, W. Baumann and W. Lang, Z. Naturforsch., Teil A, 1982, 37, 1427-1448.

7 J. Stanley, D. Smith, B. Latimer and J. P. Devlin, J. Phys. Chem., 1966, 70, 2011-2016.

8 B. Hall and J. P. Devlin, J. Phys. Chem., 1966, 71, 465-466.

9 M. Rossi and E. Haselbach, Helv. Chim. Acta, 1979, 62, 140-151.

10 M. Saheki and H. Yamada, Spectrochim. Acta, Part A, 1976, 32, $1425-1433$.

11 S. N. Cesaro, B. Martini, L. Bencivenni, M. Spoliti and M. Maltese, Spectrochim. Acta, Part A, 1980, 36, 165-170.

12 M. L. Smith and J. L. McHale, J. Phys. Chem., 1985, 89, 4002-4007.

13 J. L. McHale and M. J. Merriam, J. Phys. Chem., 1989, 93, 526-529.

14 B. M. Britt, H. B. Lueck and J. L. McHale, Chem. Phys. Lett., 1992, 190, 528-532.

15 B. M. Britt, J. L. McHale and D. M. Friedrich, J. Phys. Chem., 1995, 99, 6347-6355.

16 K. Kulinowski, I. R. Gould and A. B. Myers, J. Phys. Chem., 1995, 99, 9017-9026.

17 K. Kulinowski, I. R. Gould, N. S. Ferris and A. B. Myers, J. Phys. Chem., 1995, 99, 17715-17723.
18 N. M. Britt and J. L. McHale, Chem. Phys. Lett., 1997, 270, $551-556$

19 M. Saheki, H. Yamada, H. Yoshioka and K. Nakatsu, Acta Crystallogr., Sect. B, 1976, 32, 662-664.

20 K. Wynne, C. Galli and R. M. Hochstrasser, J. Chem. Phys., 1994, 100, 4797-4810.

21 I. V. Rubstov and K. Yoshihara, J. Phys. Chem. A, 1999, 103, 10202-10212.

22 M. J. Merriam, R. Rodriguez and J. L. McHale, J. Phys. Chem., 1987, 91, 1058-1063.

23 M. Hayashi, T.-S. Yang, J. Yu, A. Mebel and S. H. Lin, J. Phys. Chem. A, 1997, 101, 4156-4162.

24 M. Hayashi, T.-S. Yang, J. Yu, A. Mebel, R. Chang, S. H. Lin, I. V. Rubtsov and K. Yoshihara, J. Phys. Chem. A, 1998, 102, 4256-4265.

25 M.-S. Liao, Y. Lu and S. Scheiner, J. Comput. Chem., 2003, 24, 623-631.

26 K. Yamamoto, M. H. Kabir and K. Tominaga, to be published

27 M. J. Frisch, G. W. Trucks, H. B. Schlegel, G. E. Scuseria, M. A Robb, J. R. Cheeseman, J. A. Montgomery, Jr, T. Vreven, K. N. Kudin, J. C. Burant, J. M. Millam, S. S. Iyengar, J. Tomasi, V. Barone, B. Mennucci, M. Cossi, G. Scalmani, N. Rega, G. A. Petersson, H. Nakatsuji, M. Hada, M. Ehara, K. Toyota, R. Fukuda, J. Hasegawa, M. Ishida, T. Nakajima, Y. Honda, O. Kitao, H. Nakai, M. Klene, X. Li, J. E. Knox, H. P. Hratchian, J. B. Cross, C. Adamo, J. Jaramillo, R. Gomperts, R. E. Stratmann, O. Yazyev, A. J. Austin, R. Cammi, C. Pomelli, J. W. Ochterski, P. Y. Ayala, K. Morokuma, G. A. Voth, P. Salvador, J. J. Dannenberg, V. G. Zakrzewski, S. Dapprich, A. D. Daniels, M C. Strain, O. Farkas, D. K. Malick, A. D. Rabuck, K. Raghavachari, J. B. Foresman, J. V. Ortiz, Q. Cui, A. G. Baboul, S Clifford, J. Cioslowski, B. B. Stefanov, G. Liu, A. Liashenko, P. Piskorz, I. Komaromi, R. L. Martin, D. J. Fox, T. Keith, M. A. Al-Laham, C. Y. Peng, A. Nanayakkara, M. Challacombe, P. M. W. Gill, B. Johnson, W. Chen, M. W. Wong, C. Gonzalez and J. A. Pople, GAUSSIAN 03 (Revision B.4), Gaussian, Inc., Pittsburgh, PA, 2003.

28 S. F. Boys and F. Bernardi, Mol. Phys., 1970, 19, 553-566.

29 M. H. Kabir, K. Yamamoto, M. Hayashi and K. Tominaga, to be published.

30 Y. C. Shen, P. C. Upadhya, E. H. Linfield and A. G. Davies, Vib. Spectrosc., 2004, 35, 111-114.

31 D. A. McQuarrie, Statistical Mechanics, University Science Books, Sausalito, CA, 2000.

32 D. W. Oxtoby, D. Levesque and J.-J. Weis, J. Chem. Phys., 1978, 68, 5528-5533.

33 J. Stenger, D. Madsen, P. Hamm, E. T. J. Nibbering and T. Elsaesser, Phys. Rev. Lett., 2001, 87, 27401. 